\title{
Index
}

Abuse, 67, 126, 129-130

'Abused women's narratives, 62

Abuser, 67

Activism, 6-7

Age, 2

'Amore Criminale' [Criminal Love], 25

Anorexia, 83

'Antifemicide' law, 23

Audiences, 36-37

Australia, 99-100

data on child abuse in, 101-102

violence in indigenous communities in, 109-112

Australian Broadcasting Corporation

(ABC), 104, 114, 117-118

Australian media. See also Mass media coverage of child abuse, 100

landscape, 102-104

Baby Chase: How Surrogacy Is

Transforming the American

Family, The, 84

Backlash, 6-7

'Battered' woman, 87

Bias, 161

Big Little Lies, 68-71

Biological theories, 19-20

\#BlackLivesMatter movement, 36

Bondage Discipline Sadism and

Masochism (BDSM), 51

British Broadcasting Corporation

(BBC), 3, 35, 39, 233

Celebrity, 45-47

Child

exploitation, 107

grooming, 173

lack of transparency in child

protection systems, 110-112 protection, 100, 101-102

Child abuse. See also Domestic violence and abuse (DVA); Elder abuse, 99-100, 232

Australian media coverage, 100

Australian media landscape, 102-104

child abuse/protection, 126

data in Australia, 101-102

deaths, 100, 116

exposure and accountability of perpetrators in powerful positions, 116-118

harnessing public education potential of media, 118 interconnectedness of different forms of child abuse, 106-108

media reporting on child abuse and system reform, 112-116

protecting children online, 108-109

violence, objectification and resistance, 104-106

violence in indigenous communities in Australia, 109-112

Child Exploitation and Online Protection Centre (CEOP), 173

Child sexual exploitation (CSE), 173, 181-186, 234

Children's rights, 107

Class, 2

Codification, 17

Coercive control, 62-63

"Colour-blind" approach, 187

Commission for Children and Young People (CCYP), 111

Constructivism, 18-19

Context provision, 157-160 
Coronavirus pandemic [COVID-19], 6 Council of Europe Convention, 5

Counting Dead Women project, 128, 135-137

Crazy Love: A Memoir, 84

Crime

of honour, 19-22

in United Kingdom, 175-177

Criminal act, 143

Criminal Justice system, 233

Crown Prosecution Service (CPS), 174

Cultural/structural frame, 163

Custody, 71-75, 230

Daily Mail, 35, 37-38, 178-179

Daily Telegraph, The, 5, 184

Digital activism, 36

Digital connectivity, 4

Digital revolution, 2-3

[Dis]ability, 2

Discourse

analysis approach, 37, 229

practices, 20-22

Dolores Claiborne [1995], 63

Domestic abuse, 5

Domestic homicide, 37, 49

Domestic setting, 129, 142

Domestic violence (DV), 16, 82, 91, 230

case, 23-26

genre, 66-68

beyond Hollywood, 68-75

in Italian Media and Press, 18-22

literature analysis, 18-22

methodology, 22-23

narration, 23-26

postawareness, $62-64$

postfeminism, 64-65

screening in Hollywood film, 62

Domestic violence and abuse (DVA).

See also Child abuse, 1-2,

$34,80,227$

activism and backlash, 6-7 celebrity and royal connections, 45-47

context of study, 34-36

ethical reporting, 52-53

journeying, 7-9

legislative context, 4-6

methodology, 36-37

'sex game gone wrong' defence, 49-52

tabloidisation, 47-49

TED Lecture, 81-83, 89-90

transformative potential, 11

victim blaming narrative, 37-43

victims receding from view, 43-45

Elder abuse, 126

Counting Dead Women project, 135-137

critical factors, 129-130

Femicide census report, 137

future possibilities, 144-145

media reporting, 132-133

media representations and older people, 130-132

online news media reporting, 133

recommendations for improving attitudes and awareness, 143-144

theoretical perspectives, 128-129

types, 133-135

Electronic formats, 35

English Defence League, 235

Enough [film], 64-66

Episodic framing, 157-160

Ethical reporting of DVA, 52-53

Ethnicity, 2

Facebook, 35-36

Female abusers, 161

Female violence, 20-21

Female-perpetrated IPV, 161-162

Femicide, 16-17, 22, 25-26

census report, 137

in Italian Media and Press, 18-22 
Feminism, 229

Feminist jurisprudence model, 162

Femminicidio, 16-17

Film[s], 230 narratives, 67

Folk devils, South Asian Men as, 188-189

Frame, 17-18

Framing, 18

Gaslight [film], 63, 66

'Gaslighting', 5

Gender

differences in reports of domestic violence, 153-154

gender-based violence, 16-17, 20-21, 203

in United Kingdom, 175-177

Gendered DVA, 1-2

'Gendertrolling', 6-7

Genre, 66-68

Global Gender Gap Index 2020, 204-205

Gothic romance, 66-67

Grand Theft Auto 5, 4

'Grass-roots' approach, 126

Guardian, The, 45, 52, 178-179, 183-184

Hegemonic masculinity, 175

Herald Sun newspaper, 112-113, 115-116

Hollywood film, screening domestic violence in, 62

Home Box Office (HBO), 68-69

Homogenisation process, 103

Honour-based violence, 44

Illiberal democracy, 235

Independent newspaper, 34-35

Independent Press Standards

Organisation, 53

Information campaigns, 143-144

Instagram, 35-36
Institutional

elder abuse, 140-141

frame of responsibility, 163

Interconnectedness of different forms of child abuse, 106-108

Internal trafficking, 173

International Federation of Social

Workers (IFSW), 237

International Network for the Prevention of Elder Abuse (INPEA), 127

Intersectionality, 2

Intimate partner homicide (IPH), 34, $155-156$

Intimate partner violence (IPV), 16-17, $100,150-151$

Islamophobia, culture of, 176

Istanbul Convention, 5, 19-20

Italian newspapers, 20-21

Jay inquiry, 234

\#Jaztudi campaign, 201

consequences, 218-220

in Slovenia, 206-217

Journalism, 102-103

Journalistic decision-making processes, 118

Journeying, 7-9

Just Before Losing Everything [Avant que de tout perdre], 71

Labour exploitation, 174

Lecture, 91

Lesbian, gay, bisexual and transgender communities (LGBT communities), 154-155, 233

Lexis-Nexis, 177-178

Little Big Lies, 230

Localised grooming, 173

Mail on Sunday, 178-179

MailOnline, 38-41

Mainstream media, 35-36 
Mass media, 3, 236 newspapers, 91

Media, 17, 140-141 brands, 3 coverage, 17-20 discourse, 20-22 harnessing public education potential of, 118 outlets, 150

in popular culture, 2-4 responses, 217-218

Media reporting on child abuse and system reform, $112-116$

of DVA, 53

of elder abuse, 132-133

Media representation, 126-128, 130-132, 177, 180

of older people, 232

of perpetrators and victims, 160-163

Member of Order of Australia [AM], 104

\#Metoo campaign, 200-201

\#jaztudi campaign in Slovenia, 206-217

contextualising sexual violence, 201-205

media responses, 217-218

\#MeToo movement, 6, 36

Mirror newspaper, 42-43

Mistreatment, 129-130

Mobile technologies, 35

Mommy Wars: Stay-at-Home and

Career Moms Face off on

their Choices, Their Lives,

Their Families, 84

Moral panic, 172

grooming and sexual exploitation, 172-174

methodology, 177-188

race, gender, crime and moral

panics in United Kingdom, 175-177
South Asian Men as folk devils, 188-189

Muslim culture, 176

'Nagging wife' positions women, 42

Naked Truth, The, 84

National Police Chiefs Council (NPCC), 189

National Referral Mechanism, 174

National Society for the Prevention of Cruelty to Children (NSPCC), 110-111

Neglect, 126

Neo-patriarchy, 202

Neoliberal democracy, 235

News articles, 150

News media, 150

context provision and episodic vs. thematic framing, 157-160

coverage of same-sex intimate partner violence, 154-155

data source and search strategy, 151 gender differences in reports of domestic violence, 153-154 inclusion and exclusion criteria, 151 media representation of perpetrators and victims, 160-163

methodological issues, 153

newsworthiness and sensationalism, 155-157

study selection and data extraction, 152

Newspapers, 34-35

Newsworthiness, 155-157

NorVold Abuse Questionnaire (NorAQ), 203

Objectification, 104-106

Office of National Statistics (ONS), 82

Office of the Children's Commissioner for England (OCCE), 185-186

Older people, 130-132

Older women, 128 
Online news media reporting, 133

Organisational abuse. See Institutional elder abuse

Organised abuse, 118

\section{Perpetrators, 82} media representation of, 160-163

Personal Safety Survey (PSS), 101

Popular culture, 1, 63-64, 80 media in, 2-4

Portrayal of victims, 179-181

Post-socialism, 201-202

Postawareness, 62-64

Postfeminism, 64-65

Problematisation, 176

Psychological grooming, 173

Public perceptions, 34

Race, 2 in United Kingdom, 175-177

Resistance, 104-106

Ritualised abuse, 118

Royal connections, 45-47

Run for Your Life [lifetime television movie], 65

Safe Haven [film], 67-68

Safeguarding Adult Reviews in England, 142

Same-sex intimate partner violence (SSIPV), 150-151 coverage, 154-155

'Sawn-off shotgun killer', 47-48

Secondary victimisation, 41-42

Self-grooming, 173

Sensationalism, 155-157

Sensationalistic reporting style, 156-157

Separation assault, 72

'Sex game gone wrong' defence, 49-52

Sexual abuse, 212

Sexual exploitation, 172-174

Sexual harassment, 217
Sexual misadventure defence, 49-50, 51

Sexual orientation, 2

Sexual sadomasochism, 49

Sexual violence, 200-201 contextualising, 201-205

Sleeping with the Enemy [1991], 66

Slovenia, 203 \#jaztudi campaign in, 206-217

Snowball effect, 218, 219

Social factors, 91

Social media. See also Mass media communication, 4 technologies, 35-36

Sociocultural approaches, 18-19

South Asian Men as folk devils, 188-189

Speeches, 21-22

Statutory agencies, 186-188

Steiner, Leslie Morgan [advocate for women], 83-84

\#Stopthesilence, 205

Street grooming, 173

Sun, The , 178-179

Sun tabloid, 35

Sunday Times, The, 178-179

Tabloidisation, 47-49

Tabloids, 35

TED lecture, 80, 231 active viewers, 87-88

domestic violence and abuse, 81-83, 89-90

impact, 84-91

Leslie Morgan Steiner, 83-84 technical effect, 88-89

Telegraph, The, 39, 183-185

Television, 228, 236

Textual abuse, 104-105

Thematic framing, 157-160

Thriller, 66

Times, The, 43, 177-179

\#TimesUp movement, 6

Transformative potential, 11 
'Trial by media' concept, 9

Twitter, 35-36

UK national press, 35

United Kingdom, race, gender, crime and moral panics in, $175-177$

Victim blaming, 229 narrative, 37-43

Victim[s] empowerment, 65 media representation of, 160-163

Victimisation, 106

Vigilante A [movie], 67-68

Violence. See also Domestic violence (DV), 104-106

in indigenous communities in Australia, 109-112
Violence against women (VAW), 16-17, 19-20, 36-37, $139-140$

Violent intimate relationships, 16

Washington Post, The magazine, 84 What's Love Got to Do with It [1993], 63-64

Why Domestic Violence Victims Don't Leave: Crazy Love, 80

\#WhyIStayed movement, 6

Women's Aid and Refuge, 53

'Women's Views on News' online platform, 41

World Elder Abuse Awareness Day, 127, 143-144

Zero Tolerance, 53 